\title{
Clinico-Radiological Presentation of Angiocentric Neuroepithelial Tumor Associated with Pharmacoresistant Epilepsy: A Case Report
}

\author{
Anne-Laure Hermann, $M D^{1^{*}}$ (i), Christine Bulteau, MD, PhD', Marc Polivka, MD ${ }^{3}$ and Augustin Lecler, \\ $M D, P h D^{1}$ \\ ${ }^{1}$ Department of Neuroradiology, Rothschild's Foundation Hospital, Paris, France \\ ${ }^{2}$ Department of Paediatric Neurosurgery, Rothschild's Foundation Hospital, Paris, France
}

${ }^{3}$ Department of Pathology, Hôpital Lariboisière, Institute of Psychology, Sorbonne Paris Cité University, France

*Corresponding author: Anne-Laure Hermann, MD, Department of Neuroradiology, Rothschild's Foundation Hospital, 25 rue Manin, 75019 Paris, France, Tel: 00-331-48-0-364-01, Fax: 00-33-148-036-515

\section{Keywords \\ Refractory tumor-related epilepsy, Low-grade epilepsy as- sociated neuroepithelial tumors, Angiocentric glioma}

\section{Introduction}

The World Health Organization (WHO) Classification of Central Nervous System describes several types of glioneuronal tumors, which are known to induce refractory partial seizures in children and adults [1]. The most frequent ones are Dysembryoplastic Neuroepithelial Tumors (DNETs) [2], gangliogliomas and oligodendrogliomas, but other types of complex glioneuronal lesions can be observed and are described as Low-Grade Epilepsy Associated Neuroepithelial Tumors (LEAT) [3]. The angiocentric glioma, previously known as Angiocentric Neuroepithelial Tumor (ANET), is one of the rarest of glioneuronal tumors. We report this rare entity in a 10-year-old child who underwent surgery for refractory epilepsy. Despite some specific pattern on Magnetic Resonance Imaging (MRI), its appearance is not well known from the neuroradiologist community and could help clinicians achieve diagnosis and adapt patient's management.

\section{Case Description}

A 10-year-old girl with a history of slight developmental delay and epilepsy, onset 3 years prior, presented with progressively worsening, medication resistant seizure activity. Clinical evaluation showed intermittent stereotypal partial seizures with head and eye deviation to the right. EEG showed focal slow wave activity in the right precentral frontal area. Brain MRI showed a $3 \mathrm{~cm}$ right fronto-lateral expansile cortical and subcortical lesion involving mainly the precentral area. The lesion displayed a T1, T2 and FLAIR weighted-imaging (WI) hyperintense thickened cortex without any enhancement (Figure 1, arrowhead) and T2 hyperintense subcortical white matter (Figure 1B; arrow). There was no mass effect. Brain MRI was otherwise normal. The lesion was surgically removed. After five days without any crisis, the child presented recurrence of seizures that was confirmed on EEG. A second intervention guided by per-operative motricity simulation allowed to complete the resection without any damage of the motricity area.

Pathology revealed an angiocentric glioma with elongated monomorphic spindle-shaped glial cells presenting a perivascular distribution (Figure $2 \mathrm{~A}$ ), positive for glial fibrillary acidic protein GFAP (Figure 2B) and epithelial membrane antigen EMA (Figure 2C). No mitotic activity nor necrosis were identified. Final diagnosis was angiocentric glioma. During years of follow-up, neither further seizures nor symptoms of tumor recurrence were observed.

\section{Discussion}

We presented a case of angiocentric glioma, also

Citation: Hermann AL, Bulteau C, Polivka M, Lecler A (2021) Clinico-Radiological Presentation of Angiocentric Neuroepithelial Tumor Associated with Pharmacoresistant Epilepsy: A Case Report. Neurosurg Cases Rev 4:059. doi.org/10.23937/2643-4474/1710059

Accepted: February 17, 2021; Published: February 19, 2021

Copyright: (c) 2021 Hermann AL, et al. This is an open-access article distributed under the terms of the Creative Commons Attribution License, which permits unrestricted use, distribution, and reproduction in any medium, provided the original author and source are credited. 

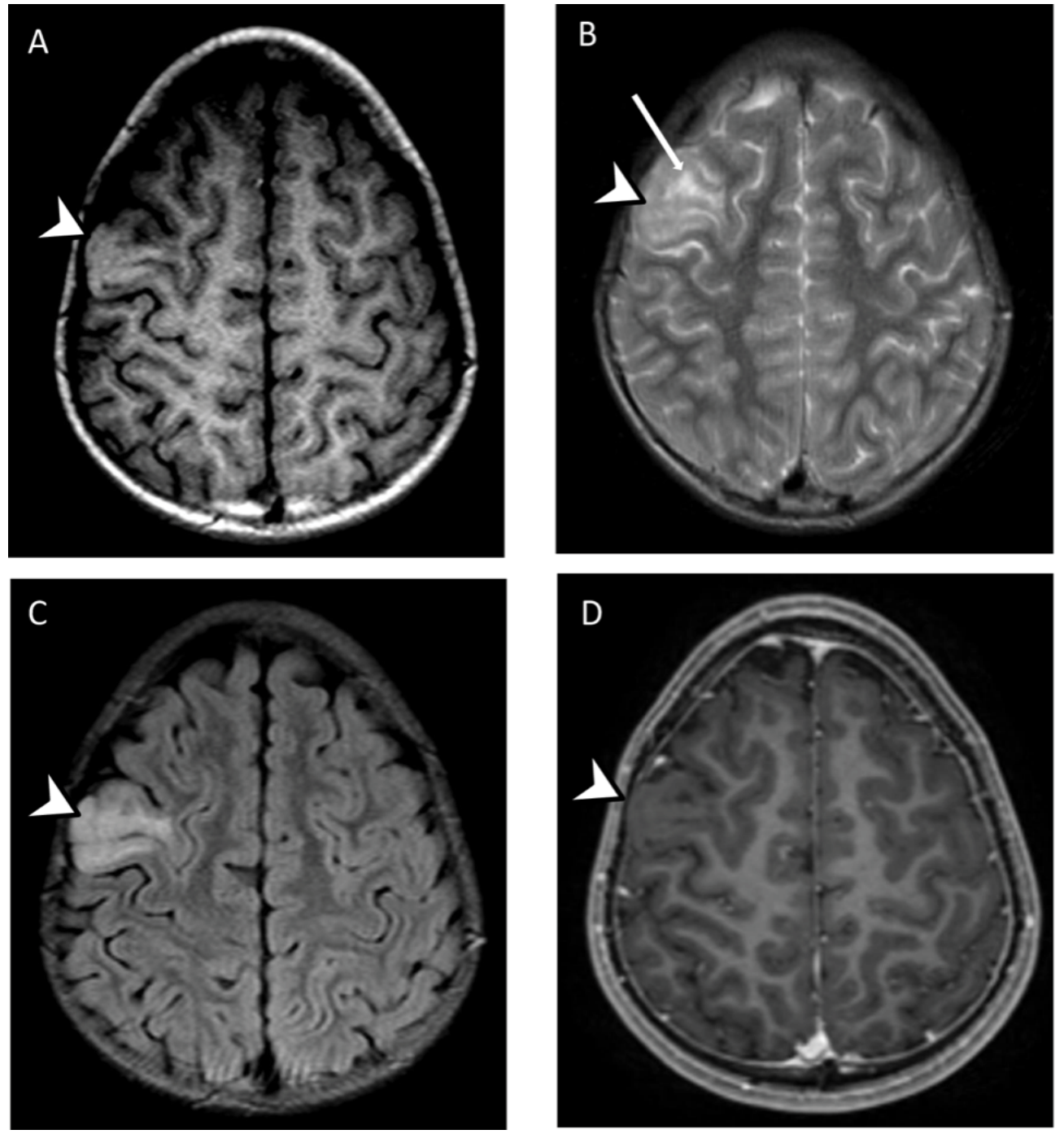

Figure 1: Axial brain MRI with T1-weighted. A) T2-weighted; B) FLAIR; C) Contrast enhanced; D) Images. It shows a T1, T2 and FLAIR hyperintense thickened cortex without any enhancement (arrowhead) and T2 hyperintense subcortical white matter (arrow). The T1 hyperintense signal has been reported as specific to angiocentric glioma.

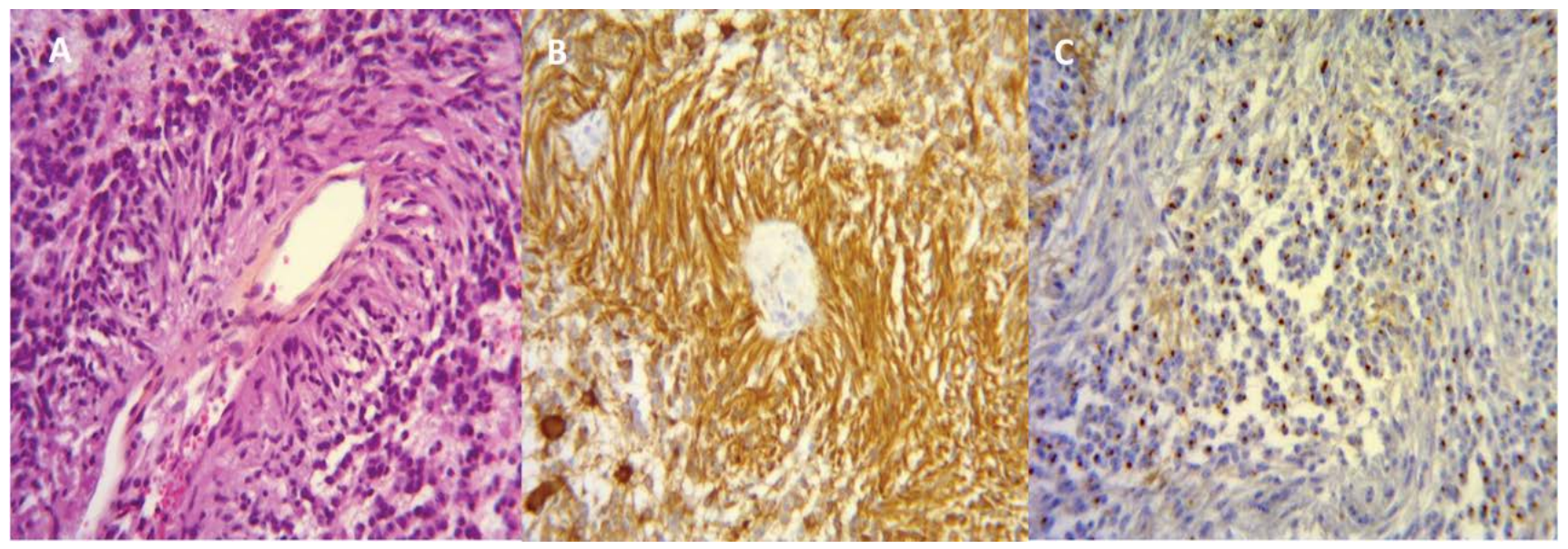

Figure 2: Microscopic views from the resection specimen using hematoxylin eosin. A) Glial fibrillary acidic protein; B) Epithelial membrane antige; C) Staining (x16 magnification). Tumoral cells presented a typically angiocentric arrangement and express glial fibrillary acidic protein and epithelial membrane antigen in a dot blot manner.

known as ANET, which has been described for the first time in 2005 and classified in 2007 as a I grade glioneuronal tumor by the WHO. This entity remains exceedingly rare with only seventy-one cases described in the literature to our knowledge [3]. Angiocentric glioma grows predominantly in supra-tentorial locations, usually in frontal and temporal cortex, but some cases have been described in mid-brain. Intractable partial epilepsy 
is the most frequent symptom. Children represent the vast majority of cases, with an age at onset of epilepsy ranging from 2 to 13 years.

Pathologically, angiocentric glioma demonstrates a pathognomonic histological pattern with angiocentric arrangement of monomorphic spindle-like, elongated and bipolar astrocytic cells forming rings around blood vessels (also called "pseudo-rosette" or "palisade-like" structures), encrusting even the vessels far beyond the main tumor mass. In immunohistochemistry, the neoplastic cells are strongly immunoreactive for Glial Fibrillary Acidic Protein (GFAP) and S100 protein, suggesting a glial origin, and also show a dot-like positivity for Epithelial Membrane Antigen (EMA), which is regarded as a signature of ependymoma. These confirm the dual astroglial-ependymal nature of the tumor. Tumor cells are immunonegative for neuronal markers as neurofilament protein and synaptophysin in contrast with the remaining neuronal component in background. Angiocentric glioma cells usually exhibit a low proliferative rate (Ki67 from 1 to $5 \%$ ), that corroborates with the slow clinical evolution observed in these tumors.

Radiologically, imaging shows angiocentric glioma as a homogeneous lesion with ill-defined margins involving both the cortex and the subcortical white matter. Affected cortical gyrus is enlarged with local effacement of adjacent cortical sulci. Computerized Tomography (CT) might allow detecting the lesion, but MRI is the modality of choice for both detection and characterization of angiocentric glioma with higher sensitivity and specificity. Both thickened cortex and subcortical white matter display a non-specific high-signal intensity on T2WI and FLAIR, with no enhancement after contrast injection. T1-WI could be non-specific with a hypointense signal but could also demonstrate characteristic pattern with a slight hyperintense rim of both the thickened cortex and subcortical white matter [4]. The T2-WI and FLAIR hyperintense "stalk like" extension perpendicular to the nearest ventricle reported in some cases as a specific appearance of the lesion could be explained by the vascular extension far beyond the main tumor mass [4]. There is usually no calcification, no hemorrhagic transformation, no diffusion-weighted imaging restriction and no enhancement. Few findings are known in the literature of histologically-typical angiocentric glioma on multiparametric imaging. Magnetic Resonance Spectroscopy profile has been reported to be non-specific, with a tendency to a mildly increased choline/creatine ratio and decreased NAA/creatine ratio in the tumor and either no or only mild increase of blood volumes and blood flows with Dynamic Susceptibility Contrast Perfusion [5].
This specific imaging pattern is very helpful to the clinician to differentiate angiocentric glioma from major differential diagnoses including focal cortical dysplasia, Dysembryo Plastic Neuroepithelial Tumor (DNET) and benign or malignant glial tumors (astrocytoma, ependymoma, oligodendroglioma, ganglioglioma). Establishing a diagnosis of angiocentric glioma with imaging helps neurosurgeons to plan their intervention and to adapt the management of the patient. Particularly, neurosurgeons should pay attention to the extension in distant regions from the original mass to ensure a total resection and avoid recurrence. Following total surgical resection using neuro-navigation methods with the help of MRI (including MRI-spectroscopy and diffusion tensor MRI), prognosis is excellent, with no tumor or seizure recurrence in the vast majority of cases [5]. Therefore, it is very important that clinicians look for this pattern and are aware of this specific finding, especially those specialized in epilepsy.

\section{Conclusion}

Angiocentric glioma is a very rare entity presenting with specific radiological and pathological features. Its imaging characteristics should be known from neuroradiologists and neurosurgeons to adapt the management of the patient.

\section{Conflict of Interest}

We have no conflict of interest.

\section{References}

1. Louis DN, Ohgaki H, Wiestler OD, Cavenee WK, Peter C Burge, et al. (2007) The 2007 WHO classification of tumours of the central nervous system. Acta Neuropathol 114: 97-109.

2. Cosson RS, Varlet $P$, Beuvon $F$, Duport CD, Devaux B, et al. (2001) Dysembryoplastic neuroepithelial tumors: CT, MR findings and imaging follow-up: A study of 53 cases. $J$ Neuroradiol 28: 230-240.

3. Giulioni M, Marucci G, Pelliccia V, Gozzo F, Barba C, et al. (2017) Epilepsy surgery of "low grade epilepsy associated neuroepithelial tumors": A retrospective nationwide Italian study. Epilepsia 58: 1832-1841.

4. Lellouch-Tubiana $A$, Boddaert $N$, Bourgeois $M$, Fohlen $M$, Jouvet A, et al. (2005) Angiocentric neuroepithelial tumor (ANET): A new epilepsy-related clinicopathological entity with distinctive MRI. Brain Pathol 15: 281-286.

5. Whitehead MT, Vezina G (2015) MR spectroscopic profile of an angiocentric glioma. Anticancer Res 35: 6267-6270.

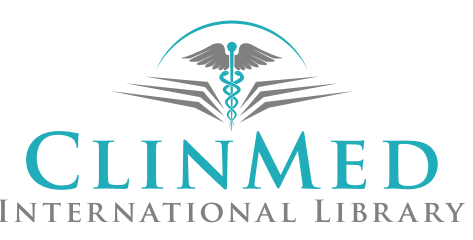

